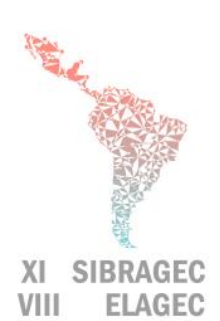

VIII ELAGEC
XI SIMPÓSIO BRASILEIRO DE GESTÃO E ECONOMIA DA CONSTRUÇÃO

VIII ENCUENTRO LATINOAMERICANO DE GESTIÓN Y ECONOMÍA DE LA CONSTRUCCIÓN

Del conocimiento a la acción: prácticas avanzadas de gestión de la producción

Londrina, Paraná, Brasil. 23 al 25 de Octubre del 2019

\title{
CAPACITACIÓN SOBRE HORMIGÓN ARMADO A OPERARIOS EN OBRA
}

\author{
LÓPEZ CANDIA, José María (1); RUIZ DÍAZ AYALA, Melanie Andrea (2); \\ GONZÁLEZ MAYA BOGADO, Jorge Nelson (3) (orientador)
}

(1) Universidad Nacional de Itapúa, Facultad de Ingeniería, +595975627188, email: josmalopezca@gmail.com, (2) Universidad Nacional de Itapúa, Facultad de Ingeniería, +595975602626, email: melaruizdiaz@hotmail.com, (3) Universidad Nacional de Itapúa, Facultad de Ingeniería, +595975633796, email: jorgegmaya@gmail.com

\begin{abstract}
Currently, it is notorious the lack of reliable information that has an inexperienced engineer regarding the process of elaboration of the concrete in situ. Therefore, the main objective of this research was the development of a physical and digital manual to improve the quality of the work carried out in a civil construction project. This paper comprises a study regarding the implementation of a training plan in civil construction work. The training plan covers the tasks associated with the formwork, assembly and elaboration of concrete, which constitutes the main material for the construction industry. The plan has been implemented by means of interviews applied to a person in charge of the project in a construction company in the city of Encarnacion, Paraguay. Then, a group of 15 workers have been trained for a period of 3 months with the aim of increase the efficiency and competence, and also, to improve the indicators of productivity and quality of the tasks mentioned above. At the end of the process, the workers have been assessed individually through written tests. The results shown an improvement of competence and productivity of the workers, as well as an increase on the quality of the works.
\end{abstract}

Keywords: construction, concrete, training, workers.

\section{INTRODUCCIÓN}

La presente investigación se realizó motivada por la importancia de conocer el elemento más importante de la construcción civil en la actualidad al cual se lo denomina Hormigón.

Si se habla de hormigón en masa se estaría hablando de la mezcla de áridos, cemento y agua en su mayor o menor proporción logrando sus principales virtudes como lo son, su gran docilidad y facilidad a adaptarse a cualquier forma, de acuerdo al encofrado utilizado.

Según JIMENEZ et al., 2000, el hormigón en masa presenta una buena resistencia a la compresión como todas las rocas que se conocen, pero ofrece escasa resistencia a la tracción. Para ello se le agregan barras de acero en zonas sometidas a tracción, llevando así el nombre de "Hormigón Armado". 
Según la norma de competencia laboral del MINISTERIO DE TRABAJO, EMPLEO Y SEGURIDAD SOCIAL de la Argentina 2010, el carpintero de obra o encofrista desarrolla procesos constructivos, interpretando las consignas derivadas de los requerimientos constructivos y haciendo uso de la información asociada, por lo que "el armador de hierros para estructuras de hormigón armado; corta, une y empalma los componentes de la armadura según las indicaciones del superior a cargo y la planilla de doblado de hierros".

Teniendo en cuenta todas estas apreciaciones se puede inferir que la capacitación permanente de operarios en obra para la elaboración del hormigón armado es necesaria e imprescindible, siguiendo las directivas e indicaciones del encargado de obra o residente, de manera a lograr la capacitación de la mano de obra y al mismo tiempo, generar seguridad no solo al accionista o inversor de la obra sino a la sociedad en general.

\section{PROBLEMA}

La ciudad de Encarnación (Paraguay) se ve afectada por grandes cambios debido a su cercanía con el río, su playa y su nuevo mote de ciudad turística, lo que genera mucho interés en cuanto a inversiones en construcciones civiles, que se presentan cotidianamente en la ciudad. Por lo tanto, el incremento de obras edilicias, despierta en todo constructor la necesidad de que su trabajo sea duradero, económico y atractivo.

Como lo decía FUENZALIDA, 2010, un alto porcentaje de los obreros de la construcción no ha recibido ningún tipo de capacitación dentro de la obra sobre las tareas que han realizado a lo largo de su vida. Significa que gran número de edificios que se construyen día a día, se llevan a cabo según conocimientos que se transmiten del abuelo al padre y luego del padre al hijo o bien, técnicas aprendidas en la práctica, siguiendo las directrices del capataz, quien les indica los procedimiento a realizar.

La mayoría de los problemas en la construcción, se ven reflejados debido a la falta de personal calificado, ya sea por tener desconocimiento en la etapa de la construcción o por negligencia, así como también por escaso control y supervisión por parte del director de obra.

Algunas de las tareas que se le designan, tomarían menos tiempo y serían de mejor calidad si pudieran aprender y ser capacitados para llevarlas a cabo.

\section{OBJETIVOS}

\subsection{General}

Capacitar a los operarios de una obra de construcción, de la ciudad de Encarnación sobre hormigón armado, a fin de obtener operarios competentes.

\subsection{Específicos}

- Identificar las habilidades, conocimientos y actitudes que posee el operario para realizar el hormigón armado, de una obra de construcción.

- Aplicar una capacitación teniendo en cuenta las deficiencias de conocimientos evidenciadas en los operarios de obra en construcción. 
- Comprobar si los operarios han adquirido los conocimientos necesarios en la capacitación, para la mejora en la realización del hormigón armado.

- Implementar una guía básica en formato físico y digital, sobre hormigón armado para el residente de obra, basado en las capacitaciones dadas.

\section{METODOLOGía}

Para la realización del proceso de investigación, primeramente se entabló contacto con una empresa del rubro de la construcción de la ciudad de Encarnación, para ofrecerles la opción de realizar charlas de capacitación sobre Hormigón Armado a sus operarios en el caso de que ellos decidan si éstos la necesiten.

La entrevista para el encargado de obra se confeccionó con la finalidad de que el mismo pudiera destacar las fortalezas o debilidades con las cuales contaban los operarios a su cargo, siempre referido a la elaboración del Hormigón Armado, aprovechando la experiencia que contaba el mismo trabajando junto con ellos, sin ser necesaria realizar una extensa entrevista individual a los operarios.

Cada clase tuvo una duración de 1 hora (50 minutos de exposición y 10 min para consultas) una vez por semana. Las mismas se llevaron a cabo durante los meses de Mayo a Agosto del 2018. Cumpliendo una totalidad de 10 charlas, distribuyéndose un total de 16 (dieciséis) módulos de capacitación realizados a 15 (quince) operarios de dicha empresa obteniendo un porcentaje mínimo individual de asistencia igual a $60 \%$ (sesenta).

Cada charla se llevó a cabo con la utilización de rotafolios hechos previamente en hojas A0, el cual contenía toda la información necesaria según los módulos correspondientes, de manera a generar un ambiente didáctico y cómodo para los operarios, teniendo en cuenta que las clases se realizaron en obra a cielo abierto.

Luego de cada módulo explicado, se les hizo llegar a los operarios un material didáctico (tríptico) referente a lo desarrollado previamente, con la idea de que les sirviese para retener todo lo explicado en dicho módulo, ejemplo de esto es apreciado en la Imágenes $1 \mathrm{y} 2$.

Al culminar el curso de capacitación, se realizó una evaluación teórica a cada operario, que consistió en la aplicación de cuestionarios de encuestas evaluativas, de manera a comprobar si han aprendido lo que se estuvo exponiendo en cada clase, verificando si se cumplió o no con el propósito establecido.

Para dejar un precedente de la capacitación realizada posteriormente se entregaron certificados a los operarios que aprobaron el módulo "Capacitación sobre encofrado, armado y elaboración de hormigón armado", obteniendo un porcentaje mínimo individual de aprobación igual a $60 \%$ (sesenta). Este certificado fue expedido por la Universidad Nacional de Itapúa, específicamente por la Facultad de Ingeniería FIUNI.

Además se elaboró un manual que dicho contenido está basado en las capacitaciones dadas a los operarios, ésta es una guía básica acreditada por el Director de la Carrera de Ingeniería Civil de la facultad y está destinada para cualquier residente cuya obra necesite la utilización de Hormigón Armado. En este se explica el proceso que se debe llevar a cabo para la correcta y calificada elaboración, encofrado y armado del Hormigón Armado. 
SIBRAGEC - ELAGEC 2019 - del 23 al 25 de Octubre - LONDRINA - PR

Aprovechando la realización del manual en forma física, nos enfocamos a darle una presentación más actualizada con la utilización de una aplicación móvil, la cual sería más accesible y de más fácil manejo pensando en la nueva generación de trabajadores relacionados al ámbito de la construcción.

\section{Imagen 1- Tríptico para los operarios de acuerdo al contenido desarrollado}

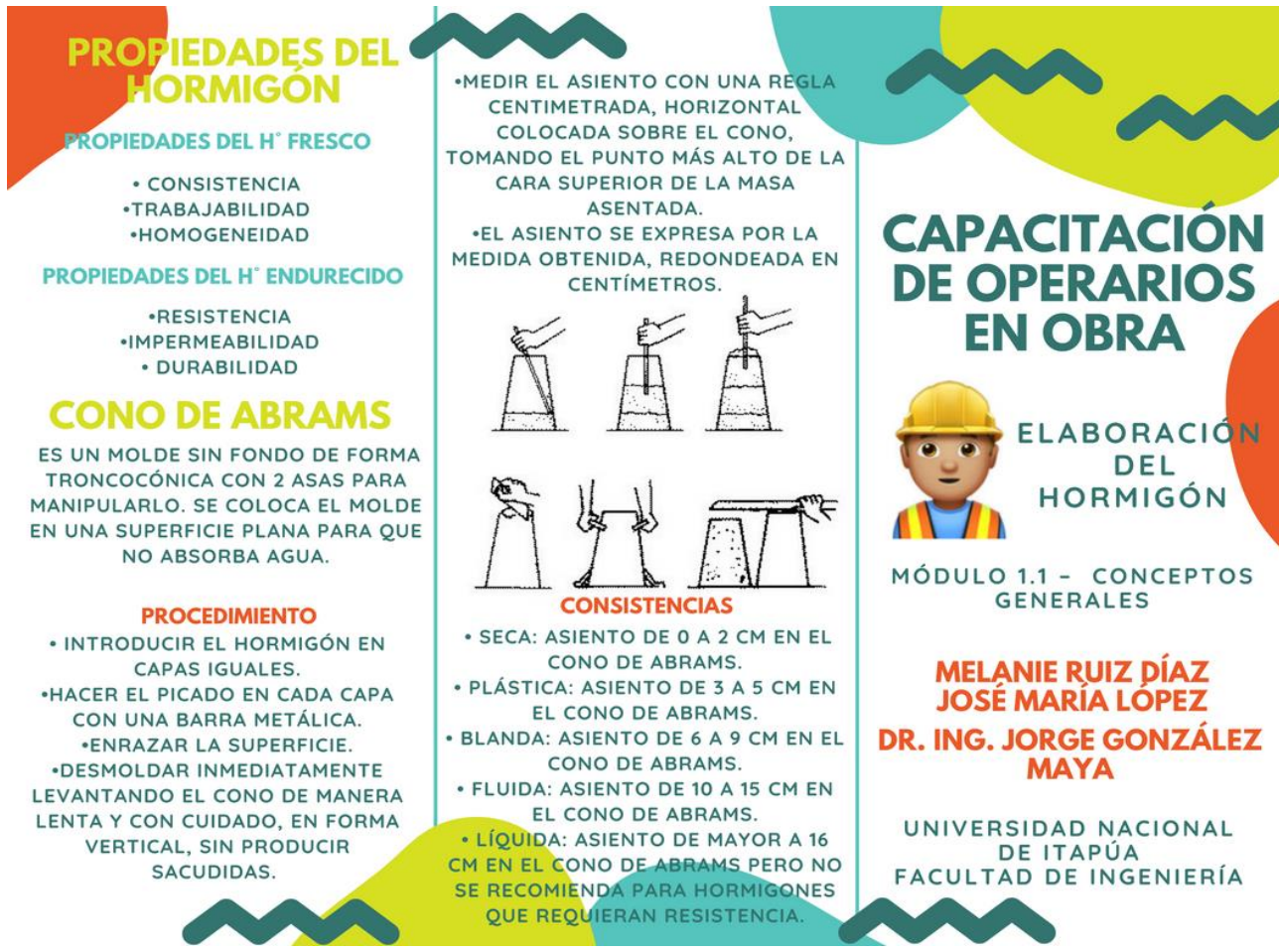

Fuente: Elaboración propia

Imagen 2- Tríptico para los operarios de acuerdo al contenido desarrollado
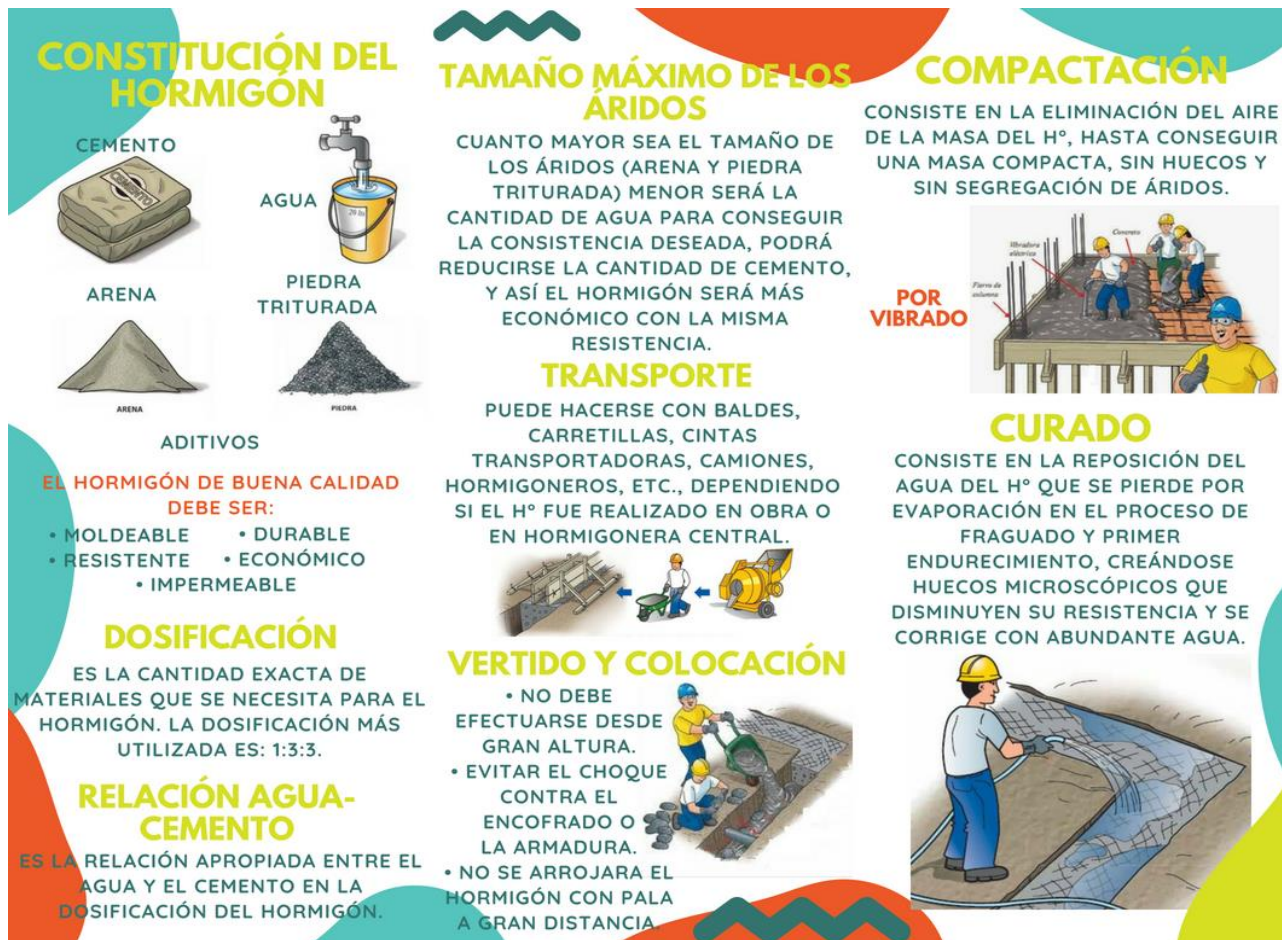

Fuente: Elaboración propia 
SIBRAGEC - ELAGEC 2019 - del 23 al 25 de Octubre - LONDRINA - PR

\section{RESULTADOS}

A partir de la entrevista realizada al encargado de obra, siendo este una persona de sexo masculino de 25 años de edad, con el título de Ingeniero civil, donde este ha expresado el bajo conocimiento que tenían los operarios acerca del hormigón armado, si bien siempre lo hacen de manera correcta en las obras, esto sería por la práctica y experiencia que han adquirido de obras anteriores pero no lo realizan con un conocimiento fundamentado.

Según expresó el encargado de obra sus operarios requerían ser capacitados, partiendo de esta preocupación se ofreció realizar charlas educativas acerca del proceso de elaboración del hormigón armado, como ya se ha mencionado en la sección de metodología.

Las encuestas evaluativas realizadas a los operarios, arrojaron resultados considerablemente buenos.

En la primera, hubo un $73 \%$ de encuestados que superaron el puntaje mínimo requerido (60\% del total) de manera correcta, mientras que solo el $27 \%$ de los mismos tuvieron puntajes menores al $60 \%$. Mientras que, en la segunda, la totalidad de los encuestados lograron sobrepasar el porcentaje mínimo para aprobar.

Tabla 1 - Resultados - Primera encuesta evaluativa

\begin{tabular}{c|c|c}
\hline Indicadores & Frecuencia & Porcentaje \\
\hline Aprobaron & 11 & 73 \\
\hline No aprobaron & 4 & 27 \\
\hline Total & 15 & 100 \\
\hline
\end{tabular}

Fuente: Elaboración propia

Interpretación: Se puede visualizar en la Tabla 1 que en un índice bastante alto, los operarios aprobaron la primera encuesta evaluativa, con un 60\% (sesenta) de exigencia, para ser exactos se trata de 11 operarios aprobados, mientras que 4 del total no llegaron al puntaje mínimo para aprobar.

Tabla 2 - Resultados- Segunda encuesta evaluativa

\begin{tabular}{c|c|c}
\hline Indicadores & Frecuencia & Porcentaje \\
\hline Aprobaron & 15 & 100 \\
\hline No aprobaron & 0 & 0 \\
\hline Total & 15 & 100 \\
\hline
\end{tabular}

Fuente: Elaboración propia

Interpretación: Se puede visualizar en la Tabla 2 que en su totalidad, es decir el 100\% de los operarios aprobaron la segunda encuesta evaluativa, con un 60\% (sesenta) de exigencia, para ser exactos se trata de 15 operarios aprobados.

Teniendo en cuenta el desempeño en ambas encuestas, 11 de los 15 operarios capacitados lograron cumplir con los requisitos necesarios para aprobar dicha 
SIBRAGEC - ELAGEC 2019 - del 23 al 25 de Octubre - LONDRINA - PR

capacitación, donde a cada uno de ellos se les otorgó un certificado de aprobación. (Imagen 3)

\section{Imagen 3- Certificado otorgado al operario}

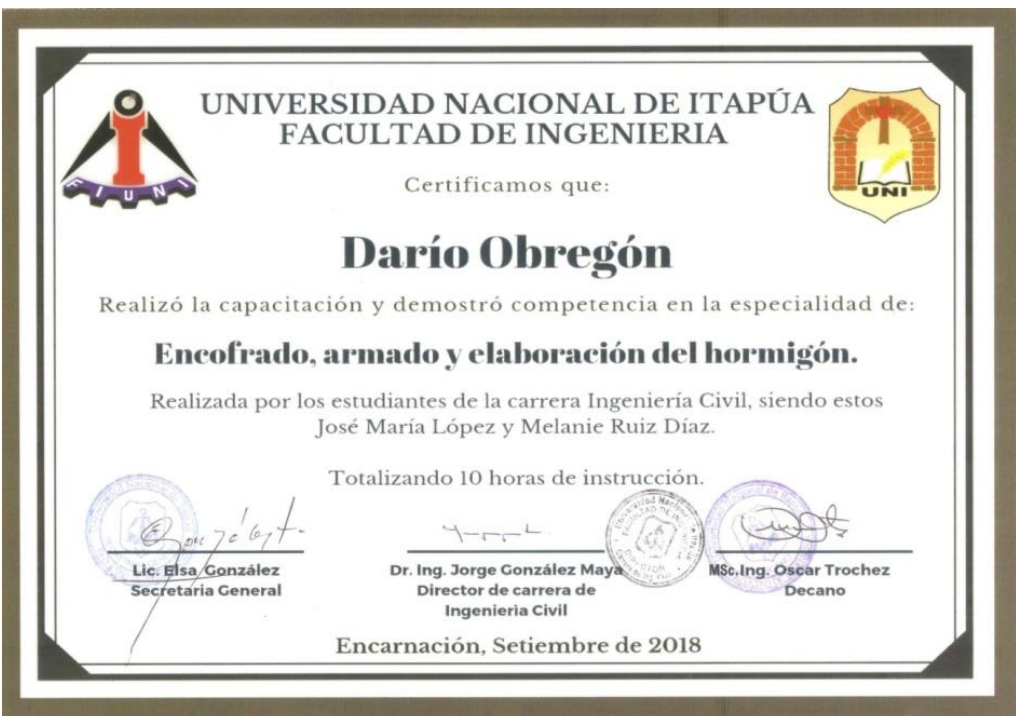

Fuente: Elaboración propia

Teniendo en cuenta la información desarrollada durante las clases, se vio conveniente la creación de un manual técnico que servirá de guía para el residente de obra y profesionales del rubro, cuando corresponda ejecutar una estructura que contenga hormigón armado.

La preparación del mismo no involucró dificultades mayores, pues la información necesaria ya fue desempeñada en las clases, solo bastó darle un formato que resultase atractivo y discreto para su utilización, el manual es mostrado en la Imagen 4.

\section{Imagen 4- Resultados- Manual para el residente de obra sobre Hormigón Armado}

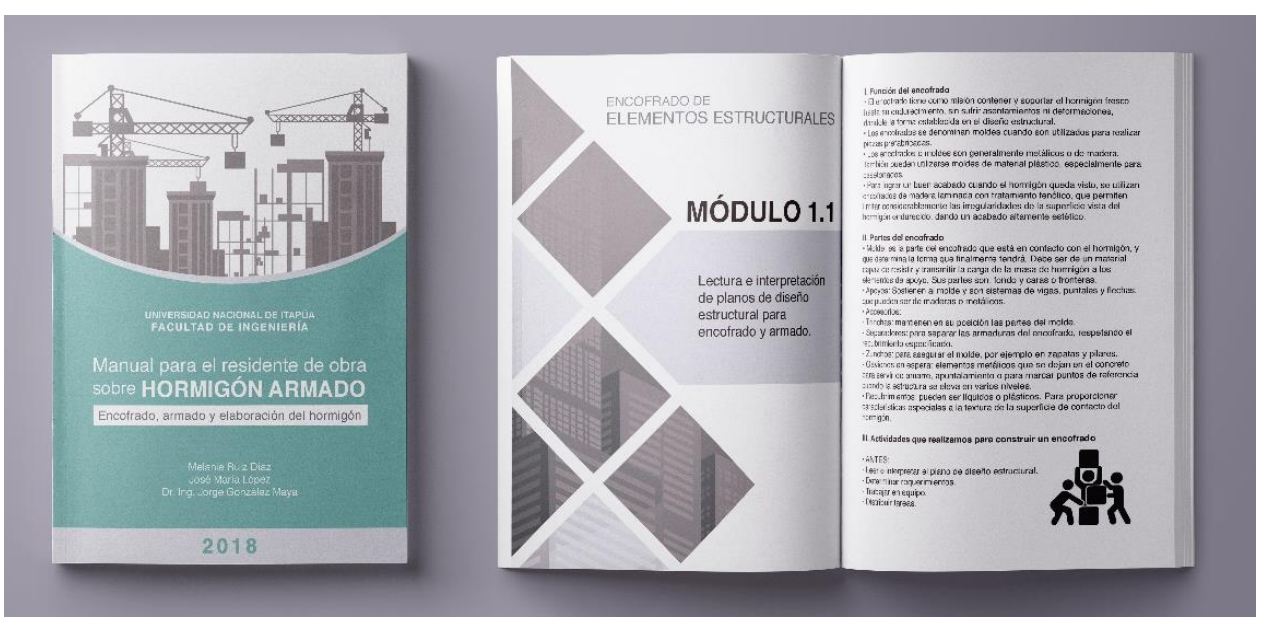

Fuente: Elaboración propia 
Aprovechando la realización del manual en forma física, se procedió a darle una presentación más actualizada con la utilización de una aplicación móvil, donde se puede acceder a través del siguiente enlace:

https://www.youtube.com/watch?v=lw-fhs_m_QI\&feature=youtu.be

En la siguiente Imagen 5 se puede apreciar el lugar de la capacitación de los operarios que fue en el propio lugar de la obra.

\section{Imagen 5 - Clase de capacitación con los operarios.}

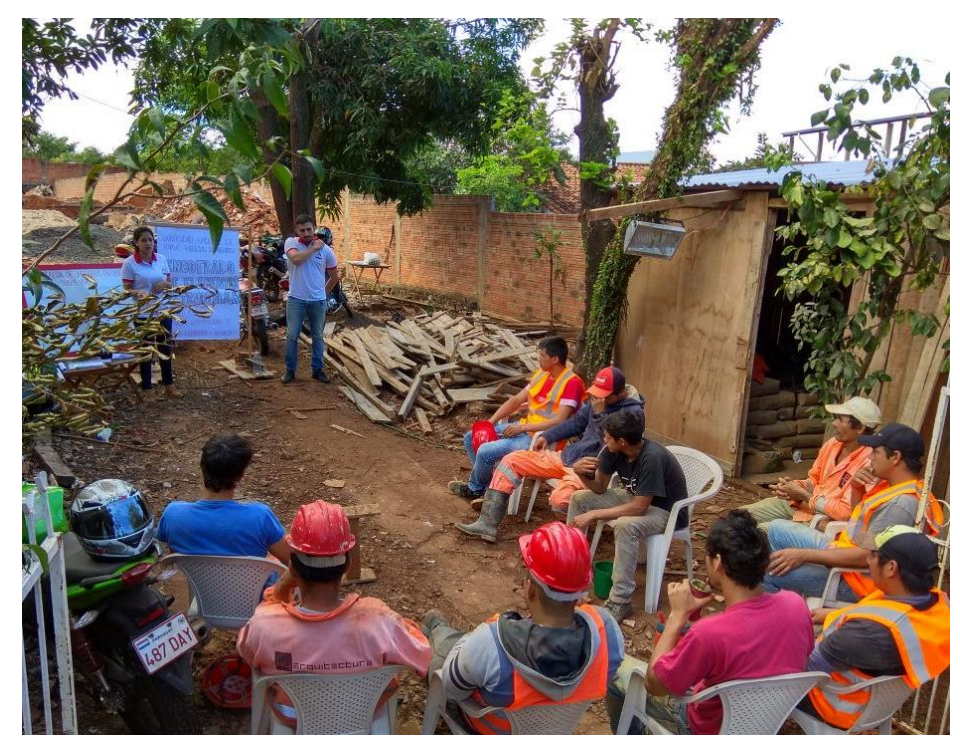

Fuente: Elaboración propia

\section{CONCLUSIONES}

-Al realizar una capacitación, siempre es necesario saber el alcance y repercusión que tuvo la misma, esto puede ser por varios medios. Lo que se puede afirmar con esto es, que del conocimiento teórico aceptable sobre Hormigón Armado que contaban los operarios, luego de las capacitaciones lograron alcanzar un considerable porcentaje, el cual habla por sí solo y explica la necesidad inminente de capacitar al operario de obra.

-Se puede decir que los operarios de obra tienen gran predisposición a capacitarse puesto que la totalidad superó el porcentaje mínimo de asistencia, lo cual estipulaba colaborar con una hora semanal para recibir las capacitaciones.

-De igual manera, la autoestima de los operarios aumentó de gran manera, ya que el curso les posicionará en mejores condiciones para un futuro empleo dentro del rubro Hormigón Armado y su elaboración, esto debido al certificado otorgado.

-Cabe resaltar que el "Manual para el residente de obra sobre HORMIGÓN ARMADO" es de libre acceso para cualquier empresa o profesional del rubro de la Construcción que esté interesado en la misma.

-La elaboración del manual en forma digital (aplicación móvil) agilizará el acceso del residente a la información requerida en el momento justo, inclusive desde su celular. 
SIBRAGEC - ELAGEC 2019 - del 23 al 25 de Octubre - LONDRINA - PR

\section{REFERENCIAS BIBLIOGRÁFICA}

ARGENTINA. $\mathrm{N}^{\circ}$ de registro: 2179466. 2010. Norma de competencia laboral Carpintero de obra fina. Argentina: Ministerio de trabajo, empleo y seguridad social, 2010. p.6

ARGENTINA. $\mathrm{N}^{\circ}$ de registro: 2179470.2010 . Norma de competencia laboral Armador de hierros para estructuras de hormigón armado. Argentina: Ministerio de trabajo, empleo y seguridad social. 2010. p.6

FUENZALIDA G., Gisela. Instituto de capacitación para el obrero de la construcción y su entorno familiar y comunitario. 2010. Memoria para optar el título de grado (Arquitecta) Facultad de arquitectura y urbanismo, Universidad de Chile, Santiago, 2010.

JIMENEZ M., Pedro, GARCIA M., Álvaro y MORAN C., Francisco. Hormigón Armado. 14.ed. Barcelona: Editorial Gustavo Gili SA, 2000. 160p. ISBN 84-252-1825-X. 